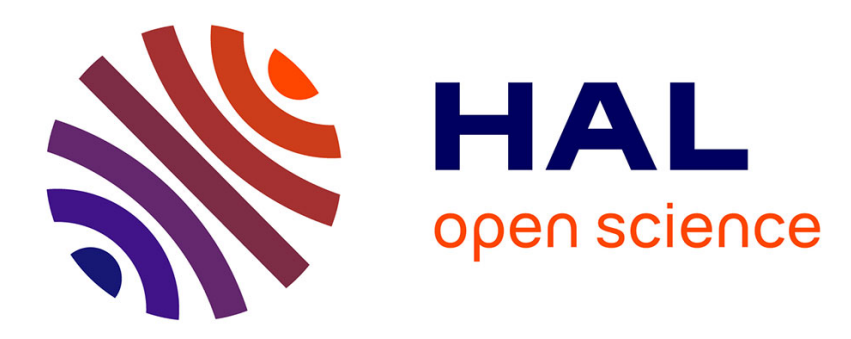

\title{
Strong maximum principles for anisotropic elliptic and parabolic equations
}

\author{
Jérôme Vétois
}

\section{To cite this version:}

Jérôme Vétois. Strong maximum principles for anisotropic elliptic and parabolic equations. Advanced Nonlinear Studies, 2012, 12 (1), pp.101-114. hal-00769037

\section{HAL Id: hal-00769037 \\ https://hal.science/hal-00769037}

Submitted on 27 Dec 2012

HAL is a multi-disciplinary open access archive for the deposit and dissemination of scientific research documents, whether they are published or not. The documents may come from teaching and research institutions in France or abroad, or from public or private research centers.
L'archive ouverte pluridisciplinaire HAL, est destinée au dépôt et à la diffusion de documents scientifiques de niveau recherche, publiés ou non, émanant des établissements d'enseignement et de recherche français ou étrangers, des laboratoires publics ou privés. 


\title{
STRONG MAXIMUM PRINCIPLES FOR ANISOTROPIC ELLIPTIC AND PARABOLIC EQUATIONS
}

\author{
JÉRÔME VÉTOIS
}

\begin{abstract}
We investigate vanishing properties of nonnegative solutions of anisotropic elliptic and parabolic equations. We describe the optimal vanishing sets, and we establish strong maximum principles.
\end{abstract}

\section{INTRODUCTION AND RESULTS}

In dimension $n \geq 2$, given $\vec{p}=\left(p_{1}, \ldots, p_{n}\right)$ with $p_{i}>1$ for $i=1, \ldots, n$, the anisotropic Laplace operator $\Delta_{\vec{p}}$ is defined by

$$
\Delta_{\vec{p}} u=\sum_{i=1}^{n} \frac{\partial}{\partial x_{i}} \nabla_{x_{i}}^{p_{i}} u
$$

where $\nabla_{x_{i}}^{p_{i}} u=\left|\partial u / \partial x_{i}\right|^{p_{i}-2} \partial u / \partial x_{i}$. We are concerned with equations of the type

$$
\Delta_{\vec{p}} u=f(x, u, \nabla u) \quad \text { in } \Omega
$$

and

$$
-\frac{\partial u}{\partial t}+\Delta_{\vec{p}} u=f(x, t, u, \nabla u) \quad \text { in } \Omega \times(0, T),
$$

where $\Omega$ is a domain in $\mathbb{R}^{n}, T$ is a positive real number, $f$ is a continuous function, and $\Delta_{\vec{p}}$ is as in (1.1). Anisotropic equations like (1.2) and (1.3) have strong physical background. They emerge, for instance, from the mathematical description of the dynamics of fluids with different conductivities in different directions. We refer to the extensive books by AntontsevDíaz-Shmarev [3] and Bear [9] for discussions in this direction. They also appear in biology, see Bendahmane-Karlsen [10] and Bendahmane-Langlais-Saad [12], as a model describing the spread of an epidemic disease in heterogeneous environments.

In this paper, we investigate strong maximum principles for anisotropic equations of the type (1.2) and (1.3). Given a subset $K$ of $\Omega$, we say that equations (1.2) and (1.3) satisfy a strong maximum principle in $K$ if any nonnegative solution which vanishes at some point in $K$ is in fact identically zero on the whole set $K$. As is well known (see, for instance, ProtterWeinberger [41]), in case of the standard harmonic and heat equations, namely in case $f=0$ and $p_{i}=2$ for all $i=1, \ldots, n$, equations (1.2) and (1.3) satisfy a strong maximum principle in the whole domain $\Omega$.

We show in this paper that in presence of anisotropy, the zeros of solutions may not spread over the whole domain $\Omega$, but they spread along directions where the anisotropic configuration is minimal. We illustrate this fact with a first example. In the anisotropic configuration

Date: April 15, 2011.

Published in Advanced Nonlinear Studies 12 (2012), no. 1, 101-114. 
$p_{1}=\cdots=p_{n-1}=p_{-}, p_{n}=p_{+}, p_{-}<p_{+}$one can check that a nonnegative stationary solution of equations (1.2) and (1.3) with $f=0$ on $\Omega=(0,+\infty)^{n-1} \times \mathbb{R}$ is given by

$$
\mathcal{U}_{\vec{p}}\left(x_{1}, \ldots, x_{n}\right)=\frac{C\left|x_{n}\right|^{p_{+} /\left(p_{+}-p_{-}\right)}}{\left(\sum_{i=1}^{n-1} x_{i}^{p_{-} /\left(p_{-}-1\right)}\right)^{\left(p_{-}-1\right) /\left(p_{+}-p_{-}\right)}},
$$

for some constant $C=C(n, \vec{p})>0$ under the assumptions that $p_{+}>p_{-}(n-2) /\left(n-1-p_{-}\right)$ and $p_{-}<n-1$. The function $\mathcal{U}_{\vec{p}}$ vanishes on the set $(0,+\infty)^{n-1} \times\{0\}$ without vanishing elsewhere in the domain. Functions of the form (1.4) were introduced, in a different context, by Giaquinta [26] and Marcellini [31]. This example can be generalized by observing that for any $C>0$ and $\varepsilon>0$, the function $\mathcal{U}_{\vec{p}}$ satisfies the inequality $\Delta_{\vec{p}} u \leq \lambda u^{p_{-}-1}$ on $\Omega=$ $(\varepsilon,+\infty)^{n-1} \times \mathbb{R}$ for $\lambda>0$ large.

In Theorem 1.1 below, we establish a strong maximum principle for elliptic inequalities of the type

$$
\Delta_{\vec{p}} u \leq f(u) \quad \text { in } \Omega .
$$

In presence of anisotropy, the vanishing sets are of the form

$$
\Omega_{0}=\left\{x \in \mathbb{R}^{n} ; \quad\left[x, \xi_{0}\right] \subset \Omega \quad \text { and } \quad x_{i}=\xi_{0, i} \quad \forall i \in \mathcal{I}_{+}\right\},
$$

for some point $\xi_{0}=\left(\xi_{0,1}, \ldots, \xi_{0, n}\right)$ in $\mathbb{R}^{n}$, where $\mathcal{I}_{+}=\left\{i \in\{1, \ldots, n\} ; \quad p_{i}>p_{-}\right\}$and $p_{-}=$ $\min \left(p_{1}, \ldots, p_{n}\right)$ is the minimum value in the anisotropic configuration. We prove our result under the assumptions that the function $f$ in the right hand sides of (1.5) is continuous, nondecreasing, and such that

$$
f(u)=\mathrm{O}\left(u^{p_{--1}}\right) \quad \text { as } u \rightarrow 0 .
$$

We let $W_{\text {loc }}^{1, \vec{p}}(\Omega)$ be the Sobolev space defined by

$$
W_{\mathrm{loc}}^{1, \vec{p}}(\Omega)=\left\{u \in L_{\mathrm{loc}}^{p_{+}}(\Omega) ; \quad \frac{\partial u}{\partial x_{i}} \in L_{\mathrm{loc}}^{p_{i}}(\Omega) \quad \forall i=1, \ldots, n\right\},
$$

where $p_{+}=\max \left(p_{1}, \ldots, p_{n}\right)$ and where, for any real number $p \geq 1, L_{\mathrm{loc}}^{p}(\Omega)$ is the space of all measurable functions on $\Omega$ which belong to $L^{p}\left(\Omega^{\prime}\right)$ for all compact subsets $\Omega^{\prime}$ of $\Omega$. We say that a function $u$ in $W_{\text {loc }}^{1, \vec{p}}(\Omega) \cap C^{0}(\Omega)$ is a (weak) solution of the inequality (1.5) if for any nonnegative smooth function $\varphi$ with compact support in $\Omega$, there holds

$$
-\int_{\Omega}\left|\frac{\partial u}{\partial x_{i}}\right|^{p_{i}-2} \frac{\partial u}{\partial x_{i}} \frac{\partial \varphi}{\partial x_{i}} d x \leq \int_{\Omega} f(u) \varphi d x .
$$

An historic reference on strong maximum principles for elliptic equations is Hopf [27]. We refer to Protter-Weinberger [41] for a reference in book form on this topic. Our first result states as follows.

Theorem 1.1. Let $\Omega$ be a nonempty domain in $\mathbb{R}^{n}$ and $f$ be a continuous nondecreasing function on $\mathbb{R}_{+}$satisfying (1.7). Let $u$ be a nonnegative solution in $W_{\text {loc }}^{1, \vec{p}}(\Omega) \cap C^{0}(\Omega)$ of inequality (1.5). If there holds $u\left(\xi_{0}\right)=0$ for some point $\xi_{0}$ in $\Omega$, then the function $u$ is identically zero on the set $\Omega_{0}$, where $\Omega_{0}$ is as in (1.6).

The vanishing sets $\Omega_{0}$ are the maximal sets on which the strong maximum principle holds true, see (1.4).

Condition (1.7) is optimal among pure nonlinearities of the type $f(u)=u^{p-1}$. Indeed, for any real number $p$ in $\left[1, p_{-}\right)$, letting $i$ be such that $p_{i}=p_{-}$, one can check that a nonnegative solution of the equation $\Delta_{\vec{p}} u=u^{p-1}$ in $\mathbb{R}^{n}$ is given by the function $\mathcal{U}_{p, p_{-}}(x)=$ 
$\left|1-C_{p, p_{-}} x_{i}\right|^{p_{-} /\left(p_{-}-p\right)}$, where $C_{p, p_{-}}=\left(p_{-}-p\right) /\left(\left(p_{-}^{p_{-}-1} p\left(p_{-}-1\right)^{2}\right)^{1 / p_{-}}\right)$. Clearly, the functions $\mathcal{U}_{p, p_{-}}$do not satisfy strong maximum principles on sets of the form (1.6).

In Theorem 1.2 below, we establish strong maximum principles for parabolic inequalities of the type

$$
-\frac{\partial u}{\partial t}+\Delta_{\vec{p}} u \leq f(u) \quad \text { in } \Omega \times(0, T) .
$$

We let $L_{\text {loc }}^{\vec{p}}\left(0, T ; W_{\text {loc }}^{1, \vec{p}}(\Omega)\right)$ be the function space defined by

$L_{\mathrm{loc}}^{\vec{p}}\left(0, T ; W_{\mathrm{loc}}^{1, \vec{p}}(\Omega)\right)=\left\{u \in L_{\mathrm{loc}}^{p_{+}}\left(0, T ; L_{\mathrm{loc}}^{p_{+}}(\Omega)\right) ; \quad \frac{\partial u}{\partial x_{i}} \in L_{\mathrm{loc}}^{p_{i}}\left(0, T ; L_{\mathrm{loc}}^{p_{i}}(\Omega)\right) \quad \forall i=1, \ldots, n\right\}$,

where $p_{+}=\max \left(p_{1}, \ldots, p_{n}\right)$ and where, for any real number $p \geq 1, L_{\mathrm{loc}}^{p}\left(0, T ; L_{\mathrm{loc}}^{p}(\Omega)\right)$ is the space of all measurable functions $u$ on $\Omega \times(0, T)$ such that $\int_{t_{1}}^{t_{2}} \int_{\Omega^{\prime}}|u|^{p} d x d t<\infty$ for all real numbers $0<t_{1}<t_{2}<T$ and all compact subsets $\Omega^{\prime}$ of $\Omega$. We say that a function $u$ in $L_{\text {loc }}^{\vec{p}}\left(0, T ; W_{\text {loc }}^{1, \vec{p}}(\Omega)\right) \cap C^{0}(\Omega \times(0, T))$ is a (weak) solution of the inequality (1.8) if for any nonnegative smooth function $\varphi$ with compact support in $\Omega \times(0, T)$, there holds

$$
\int_{0}^{T} \int_{\Omega} u \frac{\partial \varphi}{\partial t} d x d t-\int_{0}^{T} \int_{\Omega}\left|\frac{\partial u}{\partial x_{i}}\right|^{p_{i}-2} \frac{\partial u}{\partial x_{i}} \frac{\partial \varphi}{\partial x_{i}} d x d t \leq \int_{0}^{T} \int_{\Omega} f(u) \varphi d x d t .
$$

A strong maximum principle for parabolic equations involving the standard Laplace operator was obtained by Nirenberg [38]. We refer, once again, to the extensive book by ProtterWeinberger [41] on this topic. Our result states as follows.

Theorem 1.2. Let $\Omega$ be a nonempty domain in $\mathbb{R}^{n}, T$ be a positive real number, and $f$ be a continuous nondecreasing function on $\mathbb{R}_{+}$satisfying (1.7). Let $u$ be a nonnegative solution in $L_{\text {loc }}^{\vec{p}}\left(0, T ; W_{\text {loc }}^{1, \vec{p}}(\Omega)\right) \cap C^{0}(\Omega \times(0, T))$ of inequality (1.8). Assume that there holds $u\left(\xi_{0}, t_{0}\right)=0$ for some point $\xi_{0}$ in $\Omega$ and some real number $t_{0}$ in $(0, T)$. Let $\Omega_{0}$ be as in $(1.6)$. Then we get the following assertions.

(i) If $p_{-}<2$, then the function $u$ is identically zero on the set $\Omega_{0} \times\left\{t_{0}\right\}$.

(ii) If $p_{-}=2$, then the function $u$ is identically zero on the set $\Omega_{0} \times\left(0, t_{0}\right]$.

(iii) If $p_{-}>2$, then the function $u$ is identically zero on the set $\left\{\xi_{0}\right\} \times\left(0, t_{0}\right]$.

The vanishing sets in Theorem 1.2 are optimal in the sense that in case $p_{-}<2$, we get existence of solutions which extinct in finite time (see Antontsev-Shmarev [5-8]), and in case $p_{-}>2$, we get existence of solutions which vanish only on a time segment. As an example in case $p_{-}>2$, letting $i$ be such that $p_{i}=p_{-}$, one can consider the function $\mathcal{U}_{p_{-}}(x, t)=$ $\left(\left|1-C_{p_{-}} x_{i}\right|^{p_{-}} /\left(1-\left(p_{-}-2\right) t\right)\right)^{1 /\left(p_{-}-2\right)}$, where $C_{p_{-}}=\left(p_{-}-2\right) /\left(2 p_{-}^{p_{-}-1}\left(p_{-}-1\right)^{2}\right)^{1 / p_{-}}$. As is easily checked, the function $\mathcal{U}_{p_{-}}$is a nonnegative solution of the equation $\partial u / \partial t=\Delta_{\vec{p}} u$ in $\mathbb{R}^{n} \times\left(0,1 /\left(p_{-}-2\right)\right)$, and we get $\mathcal{U}_{p_{-}}(x, t)=0$ if and only if $x_{i}=1 / C_{p_{-}}$.

We refer to Antontsev-Shmarev [5-8] for several results on the existence of solutions with finite waiting time or finite extinction time and on the localization of solutions of parabolic equations like (1.3). Other possible references on anisotropic parabolic equations are Antontsev-Chipot [2], Bendahmane-Karlsen [10,11], Bendahmane-Langlais-Saad [12], and Lieberman [29]. Elliptic equations like (1.2) also received much attention in recent years. Possible references on elliptic equations like (1.2) are Alves-El Hamidi [1], Antontsev-Shmarev [4], Cianchi [13], D'Ambrosio [14], Di Castro [16], Di Castro-Montefusco [17],El Hamidi-Rakotoson [19, 20], El Hamidi-Vétois [21], Fragalà-Gazzola-Kawohl [23], Fragalà-Gazzola-Lieberman [24], García-Melián-Rossi-Sabina de Lis [25], Li [28], Lieberman [29, 30], Marcellini [32], 
Mihăilescu-Pucci-Rădulescu [34], Mihăilescu-Rădulescu-Tersian [35], Namlyeyeva-ShishkovSkrypnik [36], Skrypnik [42], Tersenov-Tersenov [43], and Vétois [45-48]. We refer to MercaldoRossi-Segura de León-Trombetti [33] for a description of the asymptotic behavior of solutions of equations like (1.2) as $p_{-} \rightarrow 1$, and we refer to Di Castro-Pérez-Llanos-Urbano [18] and Pérez-Llanos-Rossi [40] for the case $p_{-} \rightarrow \infty$, where $p_{-}=\min \left(p_{1}, \ldots, p_{n}\right)$ and $p_{+}=$ $\max \left(p_{1}, \ldots, p_{n}\right)$ are the minimum and maximum values in the anisotropic configuration.

In the isotropic configuration where $p_{i}=p$ for all $i=1, \ldots, n$, the operator (1.1) is comparable, though slightly different, to the $p$-Laplace operator $\Delta_{p}=\operatorname{div}\left(|\nabla u|^{p-2} \nabla u\right)$. We refer to Vázquez [44] where the strong maximum principle was established for elliptic equations involving the $p$-Laplace operator. As for parabolic equations involving the $p$-Laplace operator, the question of the strong maximum principle was addressed in Nazaret [37]. For more material on $p$-Laplace equations, we refer to the lecture notes by Peral [39].

We also mention the work by Fortini-Mugnai-Pucci [22] where maximum principles are established for a general class of anisotropic inequalities in divergence form, in particular in the case of variable exponents (see also Zhang [49] concerning this case).

The proofs of Theorems 1.1 and 1.2 rely on the comparison of solutions with a family of anisotropic test functions (see (2.5) and (3.3)). We prove Theorem 1.1 in Section 2, and we prove Theorem 1.2 in Section 3.

\section{Anisotropic elliptic EQUations}

In this section, we prove Theorem 1.1.

Proof of Theorem 1.1. Renumbering, if necessary, the coordinates, we may assume that there exists an index $n_{-}$such that $p_{1}=\cdots=p_{n_{-}}=p_{-}$and $p_{-}<p_{i}$ for all $i>n_{-}$. We let $\xi_{0}=\left(\xi_{0,1}, \ldots, \xi_{0, n}\right)$ be a point in $\Omega$ such that $u\left(\xi_{0}\right)=0$. We proceed by contradiction and assume that the function $u$ is not identically zero on $\Omega_{0}$, where $\Omega_{0}$ is as in (1.6). We let $P$ be the set of points $x$ in $\Omega$ such that $u(x)>0$. Since $\Omega_{0}$ is arcwise connected and since both the sets $P \cap \Omega_{0}$ and $\Omega_{0} \backslash P$ are nonempty, we get that $\partial P \cap \Omega_{0}$ is nonempty. We choose a point $\xi_{1}=\left(\xi_{1,1}, \ldots, \xi_{1, n}\right)$ in $P \cap \Omega_{0}$ such that

$$
\inf _{x \in \Omega_{0} \backslash P} \sum_{i=1}^{n_{-}}\left|x_{i}-\xi_{1, i}\right|^{\frac{p_{-}}{p_{-}}}<\inf _{x \in \partial \Omega} \sum_{i=1}^{n_{-}}\left|x_{i}-\xi_{1, i}\right|^{\frac{p_{-}}{p_{-}-1}},
$$

where, by convention, $\inf \emptyset=+\infty$. Since $P$ is open, it follows from (2.1) that there exist a positive real number $r_{0}$ and a point $\zeta_{0}=\left(\zeta_{0,1}, \ldots, \zeta_{0, n}\right)$ in $\Omega_{0} \backslash P$ such that $\zeta_{0} \in \partial B_{\xi_{1}}^{p_{-}}\left(r_{0}\right)$ and $\overline{B_{\xi_{1}}^{p_{-}}\left(r_{0}\right)} \backslash\left\{\zeta_{0}\right\} \subset P$, where

$$
B_{\xi_{1}}^{p_{-}}\left(r_{0}\right)=\left\{x \in \mathbb{R}^{n} ; \quad \sum_{i=1}^{n_{-}}\left|x_{i}-\xi_{1, i}\right|^{\frac{p_{-}}{p_{-}-1}}<r_{0} \quad \text { and } \quad x_{i}=\xi_{0, i} \quad \forall i>n_{-}\right\}
$$

and

$$
\partial B_{\xi_{1}}^{p_{-}}\left(r_{0}\right)=\left\{x \in \mathbb{R}^{n} ; \quad \sum_{i=1}^{n_{-}}\left|x_{i}-\xi_{1, i}\right|^{\frac{p_{-}}{p_{-}-1}}=r_{0} \quad \text { and } \quad x_{i}=\xi_{0, i} \quad \forall i>n_{-}\right\} .
$$

For any positive real numbers $\delta$ and $\varepsilon$, we let $A_{\xi_{1}}^{\vec{p}}\left(r_{0}, \delta, \varepsilon\right)$ be the annular set defined by

$$
A_{\xi_{1}}^{\vec{p}}\left(r_{0}, \delta, \varepsilon\right)=\left\{x \in \mathbb{R}^{n} ; \quad r_{0}-\varepsilon<\sum_{i=1}^{n} \delta^{\frac{p_{-}-p_{i}}{p_{i}-1}}\left|x_{i}-\delta \zeta_{0, i}-(1-\delta) \xi_{1, i}\right|^{\frac{p_{i}}{p_{i}-1}}<r_{0}\right\} .
$$


Since $\overline{B_{\xi_{1}}^{p_{-}}\left(r_{0}\right)} \backslash\left\{\zeta_{0}\right\} \subset P$, we get that for $\delta$ small and any $\varepsilon, A_{\xi_{1}}^{\vec{p}}\left(r_{0}, \delta, \varepsilon\right)$ is included in $\Omega$. Moreover, we get that for $\varepsilon$ fixed and $\delta$ small, the point $\zeta_{0}$ belongs to $A_{\xi_{1}}^{\vec{p}}\left(r_{0}, \delta, \varepsilon\right)$. For any positive real numbers $\lambda$ and $\delta$, we define our test function $v_{\lambda, \delta}$ on $\mathbb{R}^{n}$ by

$$
v_{\lambda, \delta}(x)=\lambda \delta\left(e^{\lambda\left(r_{0}-\sum_{i=1}^{n}\left(\lambda^{2} \delta\right)^{\frac{p_{-}-p_{i}}{p_{i}-1}} \mid x_{i}-\delta \zeta_{0, i}-(1-\delta) \xi_{1, i} \frac{p_{i}}{p_{i}-1}\right)}-1\right) .
$$

Letting $\Delta_{\vec{p}}$ be as in (1.1), we find

$$
\begin{aligned}
\Delta_{\vec{p}} v_{\lambda, \delta}(x)=\left(\lambda^{2} \delta\right)^{p_{-}-1} \sum_{i=1}^{n}( & \left.\frac{p_{i}}{p_{i}-1}\right)^{p_{i}-1} e^{\left(p_{i}-1\right) \lambda\left(r_{0}-\sum_{j=1}^{n}\left(\lambda^{2} \delta\right)^{\frac{p_{-}-p_{j}}{p_{j}-1}}\left|x_{j}-\delta \zeta_{0, j}-(1-\delta) \xi_{1, j}\right|^{\frac{p_{j}}{p_{j}-1}}\right)} \\
& \times\left(p_{i} \lambda^{\frac{2 p_{-}-p_{i}-1}{p_{i}-1}} \delta^{\frac{p_{-}-p_{i}}{p_{i}-1}} \mid x_{i}-\delta \zeta_{0, i}-(1-\delta) \xi_{1, i} i^{\frac{p_{i}}{p_{i}-1}}-1\right) .
\end{aligned}
$$

For any point $x$ in $A_{\xi_{1}}^{\vec{p}}\left(r_{0}, \lambda^{2} \delta, \varepsilon\right)$, by (1.7), (2.5), and (2.6), we get

$$
\begin{aligned}
- & \Delta_{\vec{p}} v_{\lambda, \delta}(x)+f\left(v_{\lambda, \delta}(x)\right) \\
& \leq(\lambda \delta)^{p_{-}-1}\left(\sum_{i=1}^{n}\left(\frac{p_{i}}{p_{i}-1}\right)^{p_{i}-1} \lambda^{p_{-}-1} e^{\left(p_{i}-1\right) \lambda \varepsilon}-\frac{p_{-}^{p_{-}} \lambda^{p_{-}}\left(r_{0}-\varepsilon\right)}{\left(p_{-}-1\right)^{p_{-}-1}}+C\left(e^{\lambda \varepsilon}-1\right)^{p_{-}-1}\right)
\end{aligned}
$$

when $\lambda \delta$ and $\lambda \varepsilon$ are small, for some positive constant $C$ independent of $\lambda, \delta, \varepsilon$, and $x$. Choosing $\lambda$ large enough so that

$$
\lambda>\frac{\left(p_{-}-1\right)^{p_{-}-1}}{p_{-}^{p_{-}} r_{0}} \sum_{i=1}^{n}\left(\frac{p_{i}}{p_{i}-1}\right)^{p_{i}-1},
$$

and then, choosing $\delta$ and $\varepsilon$ small, it follows from (2.7) that $v_{\lambda, \delta}$ is a $C^{1}$-solution of the inequality

$$
-\Delta_{\vec{p}} v_{\lambda, \delta}+f\left(v_{\lambda, \delta}\right)<0 \quad \text { in } A_{\xi_{1}}^{\vec{p}}\left(r_{0}, \lambda^{2} \delta, \varepsilon\right)
$$

where $A_{\xi_{1}}^{\vec{p}}\left(r_{0}, \lambda^{2} \delta, \varepsilon\right)$ is as in (2.4). We let $\partial_{1} A_{\xi_{1}}^{\vec{p}}\left(r_{0}, \lambda^{2} \delta, \varepsilon\right)$ and $\partial_{2} A_{\xi_{1}}^{\vec{p}}\left(r_{0}, \lambda^{2} \delta, \varepsilon\right)$ stand for the respective interior and exterior boundaries of the annular set $A_{\xi_{1}}^{\vec{p}}\left(r_{0}, \lambda^{2} \delta, \varepsilon\right)$. Since the function $u$ is positive on $B_{\xi_{1}}^{p_{-}}\left(r_{0}\right)$, by continuity, we get the existence of a positive constant $C_{\varepsilon}$ such that $u>C_{\varepsilon}$ on $B_{\xi_{1}}^{p_{-}}\left(r_{0}-\varepsilon\right)$, where $B_{\xi_{1}}^{p_{-}}\left(r_{0}-\varepsilon\right)$ is as in (2.2). Still by continuity of $u$, it follows that $u \geq C_{\varepsilon}$ on $\partial_{1} A_{\xi_{1}}^{\vec{p}}\left(r_{0}, \lambda^{2} \delta, \varepsilon\right)$ for $\delta$ small. Since $v_{\lambda, \delta}=\lambda \delta\left(e^{\lambda \varepsilon}-1\right)$ on $\partial_{1} A_{\xi_{1}}^{\vec{p}}\left(r_{0}, \lambda^{2} \delta, \varepsilon\right)$ and $v_{\lambda, \delta}=0$ on $\partial_{2} A_{\xi_{1}}^{\vec{p}}\left(r_{0}, \lambda^{2} \delta, \varepsilon\right)$, we then get $v_{\lambda, \delta} \leq u$ on $\partial A_{\xi_{1}}^{\vec{p}}\left(r_{0}, \lambda^{2} \delta, \varepsilon\right)$ for $\delta$ small. In particular, there holds $\left(v_{\lambda, \delta}-u\right)_{+}=0$ on $\partial A_{\xi_{1}}^{\vec{p}}\left(r_{0}, \lambda^{2} \delta, \varepsilon\right)$, where $\left(v_{\lambda, \delta}-u\right)_{+}=\max \left(v_{\lambda, \delta}-u, 0\right)$. Testing (1.5) and (2.8) against $\left(v_{\lambda, \delta}-u\right)_{+}$and integrating by parts on $A_{\xi_{1}}^{\vec{p}}\left(r_{0}, \lambda^{2} \delta, \varepsilon\right)$, we then get

$$
\begin{aligned}
\sum_{i=1}^{n} \int_{W_{\lambda, \delta, \varepsilon}}\left(\left|\frac{\partial v_{\lambda, \delta}}{\partial x_{i}}\right|^{p_{i}-2} \frac{\partial v_{\lambda, \delta}}{\partial x_{i}}-\left|\frac{\partial u}{\partial x_{i}}\right|^{p_{i}-2}\right. & \left.\frac{\partial u}{\partial x_{i}}\right)\left(\frac{\partial v_{\lambda, \delta}}{\partial x_{i}}-\frac{\partial u}{\partial x_{i}}\right) d x \\
& +\int_{W_{\lambda, \delta, \varepsilon}}\left(f\left(v_{\lambda, \delta}\right)-f(u)\right)\left(v_{\lambda, \delta}-u\right) d x \leq 0
\end{aligned}
$$

where

$$
W_{\lambda, \delta, \varepsilon}=\left\{x \in A_{\xi_{1}}^{\vec{p}}\left(r_{0}, \lambda^{2} \delta, \varepsilon\right) ; \quad v_{\lambda, \delta}(x)>u(x)\right\}
$$


Since the function $f$ is nondecreasing, it follows from (2.9) that

$$
\sum_{i=1}^{n} \int_{W_{\lambda, \delta, \varepsilon}}\left(\left|\frac{\partial v_{\lambda, \delta}}{\partial x_{i}}\right|^{p_{i}-2} \frac{\partial v_{\lambda, \delta}}{\partial x_{i}}-\left|\frac{\partial u}{\partial x_{i}}\right|^{p_{i}-2} \frac{\partial u}{\partial x_{i}}\right)\left(\frac{\partial v_{\lambda, \delta}}{\partial x_{i}}-\frac{\partial u}{\partial x_{i}}\right) d x=0
$$

and thus that $\nabla u=\nabla v_{\lambda, \delta}$ almost everywhere in $W_{\lambda, \delta, \varepsilon}$. Since $W_{\lambda, \delta, \varepsilon}$ is open, we then get that the function $v_{\lambda, \delta}-u$ is constant in $W_{\lambda, \delta, \varepsilon}$. By continuity of $u$ and $v_{\lambda, \delta}$, it follows that $\left|W_{\lambda, \delta, \varepsilon}\right|=0$, i.e. $v_{\lambda, \delta} \leq u$ in $A_{\xi_{1}}^{\vec{p}}\left(r_{0}, \lambda^{2} \delta, \varepsilon\right)$. In particular, we get $u\left(\zeta_{0}\right) \geq v_{\lambda, \delta}\left(\zeta_{0}\right)>0$. There is a contradiction. This ends the proof of Theorem 1.1.

\section{Anisotropic parabolic equations}

This section is devoted to the proof of Theorem 1.2. Renumbering, if necessary, the coordinates, we may assume in what follows that there exists an index $n_{-}$such that $p_{1}=\cdots=$ $p_{n_{-}}=p_{-}$and $p_{-}<p_{i}$ for all $i>n_{-}$. For any positive real numbers $\mu, r$, and any point $(\xi, t)$ in $\mathbb{R}^{n} \times \mathbb{R}_{+}$, we define the sets $B_{(\xi, t)}^{p_{-}}(\mu, r)$ and $\partial B_{(\xi, t)}^{p_{-}}(\mu, r)$ by

$$
B_{(\xi, t)}^{p_{-}}(\mu, r)=\left\{(x, s) \in \mathbb{R}^{n} \times \mathbb{R}_{+} ; \sum_{i=1}^{n_{-}}\left|x_{i}-\xi_{i}\right|^{\frac{p_{-}}{p_{-}-1}}+\mu|s-t|^{\frac{p_{-}}{p_{-}-1}}<r \text { and } x_{i}=\xi_{i} \forall i>n_{-}\right\}
$$

and

$$
\partial B_{(\xi, t)}^{p_{-}}(\mu, r)=\left\{(x, s) \in \mathbb{R}^{n} \times \mathbb{R}_{+} ; \sum_{i=1}^{n_{-}}\left|x_{i}-\xi_{i}\right|^{\frac{p_{-}}{p_{-}-1}}+\mu|s-t|^{\frac{p_{-}}{p_{-}-1}}=r \text { and } x_{i}=\xi_{i} \forall i>n_{-}\right\} .
$$

As a preliminary step in the proof of Theorem 1.2, we prove the following lemma.

Lemma 3.1. Let $\Omega, T, f$, and $u$ be as in Theorem 1.2. Let $\mu$ be a positive real number. Assume that there exist a positive real number $r_{0}$ and two points $\left(\xi_{0}, t_{0}\right)$ and $\left(\xi_{1}, t_{1}\right)$ in $\Omega \times(0, T)$ such that $u\left(\xi_{0}, t_{0}\right)=0,\left(\xi_{0}, t_{0}\right) \in \partial B_{\left(\xi_{1}, t_{1}\right)}^{p_{-}}\left(\mu, r_{0}\right), B_{\left(\xi_{1}, t_{1}\right)}^{p_{-}}\left(\mu, r_{0}\right) \subset \Omega_{0} \times(0, T)$, and $u(x, t)>0$ for all points $(x, t)$ in $B_{\left(\xi_{1}, t_{1}\right)}^{p_{-}}\left(\mu, r_{0}\right) \backslash\left\{\left(\xi_{0}, t_{0}\right)\right\}$, where $\Omega_{0}$ is as in $(1.6), B_{\left(\xi_{1}, t_{1}\right)}^{p_{-}}\left(\mu, r_{0}\right)$ is as in (3.1), and $\partial B_{\left(\xi_{1}, t_{1}\right)}^{p_{-}}\left(\mu, r_{0}\right)$ is as in (3.2). Then we get the following assertions.

(i) If $p_{-} \leq 2$, then $\xi_{0}=\xi_{1}$.

(ii) If $p_{-}=2$ and $\mu>\frac{1}{4 r_{0}}\left(\sum_{i=1}^{n}\left(\frac{p_{i}}{p_{i}-1}\right)^{p_{i}-1}\right)^{2}$, then $t_{0}=t_{1}-\sqrt{r_{0} / \mu}$.

(iii) If $p_{-}>2$, then $t_{0} \leq t_{1}$.

Proof of Lemma 3.1. We proceed by contradiction and assume that $\xi_{0} \neq \xi_{1}$ if $p_{-}<2$, either $\xi_{0} \neq \xi_{1}$ or $t_{0}>t_{1}$ if $p_{-}=2$, and $t_{0}>t_{1}$ if $p_{-}>2$. Moreover, decreasing, if necessary, the real number $r_{0}$, we may assume that $u(x, t)>0$ for all points $(x, t)$ on $\partial B_{\left(\xi_{1}, t_{1}\right)}^{p_{-}}\left(\mu, r_{0}\right) \backslash\left\{\left(\xi_{0}, t_{0}\right)\right\}$, where $\partial B_{\left(\xi_{1}, t_{1}\right)}^{p_{-}}\left(\mu, r_{0}\right)$ is as in (3.2). For any positive real numbers $\lambda, \mu$, and $\delta$, we define our test function $v_{\lambda, \mu, \delta}$ on $\mathbb{R}^{n} \times \mathbb{R}_{+}$by

$$
v_{\lambda, \mu, \delta}(x)=\lambda \delta\left(e^{\lambda\left(r_{0}-\sum_{i=1}^{n}\left(\lambda^{2} \delta\right)^{\frac{p_{-}-p_{i}}{p_{i}-1}}\left|x_{i}-\delta \xi_{0, i}-(1-\delta) \xi_{1, i}\right|^{\frac{p_{i}}{p_{i}-1}}-\mu\left|t-\delta t_{0}-(1-\delta) t_{1}\right|^{\frac{p_{-}}{p_{-}-1}}\right)}-1\right),
$$

where $\xi_{0}=\left(\xi_{0,1}, \ldots, \xi_{0, n}\right)$ and $\xi_{1}=\left(\xi_{1,1}, \ldots, \xi_{1, n}\right)$. We find

$$
\begin{aligned}
\frac{\partial v_{\lambda, \mu, \delta}}{\partial t}(x, t)=\frac{p_{-}}{p_{-}-1} \mu \lambda^{2} \delta e^{\lambda\left(r_{0}-\sum_{i=1}^{n}\left(\lambda^{2} \delta\right)^{\frac{p_{-}-p_{i}}{p_{i}-1}}\left|x_{i}-\delta \xi_{0, i}-(1-\delta) \xi_{1, i}\right|^{\frac{p_{i}}{p_{i}-1}}-\mu\left|t-\delta t_{0}-(1-\delta) t_{1}\right|^{\frac{p_{-}}{p_{-}-1}}\right)} \\
\times\left|\delta t_{0}+(1-\delta) t_{1}-t\right|^{\frac{2-p_{-}}{p_{-}-1}}\left(\delta t_{0}+(1-\delta) t_{1}-t\right) .
\end{aligned}
$$




$$
\begin{aligned}
& \Delta_{\vec{p}} v_{\lambda, \mu, \delta}(x, t)=\left(\lambda^{2} \delta\right)^{p_{-}-1} \sum_{i=1}^{n}\left(\frac{p_{i}}{p_{i}-1}\right)^{p_{i}-1} \\
& \times e^{\left(p_{i}-1\right) \lambda\left(r_{0}-\sum_{j=1}^{n}\left(\lambda^{2} \delta\right)^{\frac{p_{-}-p_{j}}{p_{j}-1}}\left|x_{j}-\delta \xi_{0, j}-(1-\delta) \xi_{1, j}\right|^{\frac{p_{j}}{p_{j}-1}}-\mu\left|t-\delta t_{0}-(1-\delta) t_{1}\right|^{\frac{p_{-}}{p_{-}-1}}\right)} \\
& \times\left(p_{i} \lambda^{\frac{2 p_{-}-p_{i}-1}{p_{i}-1}} \delta^{\frac{p_{-}-p_{i}}{p_{i}-1}}\left|x_{i}-\delta \xi_{0, i}-(1-\delta) \xi_{1, i}\right|^{\frac{p_{i}}{p_{i}-1}}-1\right) .
\end{aligned}
$$

As is easily seen, for $\delta$ small, for any $i=1, \ldots, n$ and any point $(x, t)$ in $\mathbb{R}^{n} \times \mathbb{R}_{+}$, there holds

$$
\begin{aligned}
& || x_{i}-\delta \xi_{0, i}-\left.(1-\delta) \xi_{1, i}\right|^{\frac{p_{i}}{p_{i}-1}}-\left|\xi_{0, i}-\xi_{1, i}\right|^{\frac{p_{i}}{p_{i}-1}} \mid \\
& \leq C\left(\left|\xi_{0, i}-\xi_{1, i}\right|^{\frac{1}{p_{i}-1}}\left|x_{i}-\xi_{0, i}\right|+\left|x_{i}-\xi_{0, i}\right|^{\frac{p_{i}}{p_{i}-1}}+\delta\left|\xi_{0, i}-\xi_{1, i}\right|^{\frac{p_{i}}{p_{i}-1}}\right) \\
& \left.|| t-\delta t_{0}-\left.(1-\delta) t_{1}\right|^{\frac{p_{-}}{p_{-}-1}}-\left.\left|t_{0}-t_{1}\right|^{\frac{p_{-}}{p_{-}-1}}\right|^{\frac{p_{-}}{p_{-}}}+\delta\left|t_{0}-t_{1}\right|^{\frac{p_{-}}{p_{-}-1}}\right) \\
& \leq C\left(\left|t_{0}-t_{1}\right|^{\frac{1}{p_{-}-1}}\left|t-t_{0}\right|+\left|t-t_{0}\right|^{\frac{p_{-}}{2}}-\right.
\end{aligned}
$$

and

$$
\begin{aligned}
& || t-\delta t_{0}-\left.(1-\delta) t_{1}\right|^{\frac{2-p_{-}}{p_{-}-1}}\left(t-\delta t_{0}-(1-\delta) t_{1}\right)-\left|t_{0}-t_{1}\right|^{\frac{2-p_{-}}{p_{-}-1}}\left(t_{0}-t_{1}\right) \mid \\
& \quad \leq \begin{cases}C\left(\left|t_{0}-t_{1}\right|^{\frac{2-p_{-}}{p_{-}}}\left|t-t_{0}\right|+\left|t-t_{0}\right|^{\frac{1}{p_{-}-1}}+\delta\left|t_{0}-t_{1}\right|^{\frac{1}{p_{-}-1}}\right) & \text { if } p_{-} \leq 2 \\
C\left(\left|t-t_{0}\right|^{\frac{1}{p_{-}-1}}+\delta^{\frac{1}{p_{-}-1}}\left|t_{0}-t_{1}\right|^{\frac{1}{p_{-}-1}}\right) & \text { if } p_{-}>2\end{cases}
\end{aligned}
$$

for some positive constant $C$ independent of $\delta, x$, and $t$. For any positive real numbers $\mu, \delta$, and $\varepsilon$, we define the ellipsoidal ball $B_{\left(\xi_{0}, t_{0}\right)}^{\vec{p}}(\mu, \delta, \varepsilon)$ by

$$
B_{\left(\xi_{0}, t_{0}\right)}^{\vec{p}}(\mu, \delta, \varepsilon)=\left\{(x, s) \in \mathbb{R}^{n} \times \mathbb{R}_{+} ; \quad \sum_{i=1}^{n} \delta^{\frac{p_{-}-p_{i}}{p_{i}-1}}\left|x_{i}-\xi_{0, i}\right|^{\frac{p_{i}}{p_{i}-1}}+\mu\left|t-t_{0}\right|^{\frac{p_{-}}{p_{-}-1}}<\varepsilon\right\}
$$

Clearly, for $\mu$ large and for $\delta$ and $\varepsilon$ small, $B_{\left(\xi_{0}, t_{0}\right)}^{\vec{p}}(\mu, \delta, \varepsilon)$ is included in $\Omega \times(0, T)$. For any positive real numbers $\lambda, \mu, \delta, \varepsilon$, and any point $(x, t)$ in $B_{\left(\xi_{0}, t_{0}\right)}^{\vec{p}}\left(\mu, \lambda^{2} \delta, \varepsilon\right)$, by (1.7), (3.3)-(3.8), 
and since $\left(\xi_{0}, t_{0}\right) \in \partial B_{\left(\xi_{1}, t_{1}\right)}^{p_{-}}\left(\mu, r_{0}\right)$, we get

$$
\begin{aligned}
& \frac{\partial v_{\lambda, \mu, \delta}}{\partial t}(x, t) \leq \frac{p_{-}}{p_{-}-1} \mu \lambda^{2} \delta \\
& \quad \times\left\{\begin{array}{cc}
e^{C \lambda(\mu+1)\left(\varepsilon^{\frac{p_{-}-1}{p_{-}}}+\delta\right)}\left(\left|t_{1}-t_{0}\right|^{\frac{2-p_{-}}{p_{-}-1}}\left(t_{1}-t_{0}\right)+C\left(\varepsilon^{\frac{p_{-}-1}{p_{-}}}+\delta\right)\right) & \text { if } p_{-} \leq 2 \text { and } t_{0} \leq t_{1} \\
e^{-C \lambda(\mu+1)\left(\varepsilon^{\frac{p_{-}-1}{p_{-}}}+\delta\right)}\left|t_{1}-t_{0}\right|^{\frac{2-p_{-}}{p_{-}-1}}\left(t_{1}-t_{0}\right) & \text { if } p_{-} \leq 2 \text { and } t_{0}>t_{1} \\
+C e^{C \lambda(\mu+1)\left(\varepsilon^{\frac{p_{-}-1}{p_{-}}}+\delta\right)}\left(\varepsilon^{\frac{p_{-}-1}{p_{-}}}+\delta\right) & \\
e^{-C \lambda(\mu+1)\left(\varepsilon^{\frac{p_{-}-1}{p_{-}}}+\delta\right)}\left|t_{1}-t_{0}\right|^{\frac{2-p_{-}-1}{p_{-}}}\left(t_{1}-t_{0}\right) \\
+C e^{C \lambda(\mu+1)\left(\varepsilon^{\frac{p_{-}-1}{p_{-}}}+\delta\right)}\left(\varepsilon^{\frac{1}{p_{-}}}+\delta^{\frac{1}{p_{-}-1}}\right) & \text { if } p_{-}>2 \text { and } t_{0}>t_{1}
\end{array}\right.
\end{aligned}
$$

and

$$
\begin{aligned}
& -\Delta_{\vec{p}} v_{\lambda, \mu, \delta}(x, t)+f\left(v_{\lambda, \mu, \delta}(x, t)\right) \leq(\lambda \delta)^{p_{-}-1}\left(\sum_{i=1}^{n}\left(\frac{p_{i}}{p_{i}-1}\right)^{p_{i}-1} \lambda^{p_{-}-1} e^{\left(p_{i}-1\right) C \lambda(\mu+1)\left(\varepsilon^{\frac{p_{-}-1}{p_{-}}}+\delta\right)}\right. \\
& -\frac{p_{-}^{p_{-}} \lambda^{p_{-}}}{\left(p_{-}-1\right)^{p_{-}-1}} e^{-\left(p_{+}-1\right) C \lambda(\mu+1)\left(\varepsilon^{\frac{p_{-}-1}{p_{-}}}+\delta\right)}\left(r_{0}-\mu\left|t_{1}-t_{0}\right|^{\frac{p_{-}}{p_{-}-1}}\right) \\
& \left.+C e^{C \lambda(\mu+1)\left(\varepsilon^{\frac{p_{-}-1}{p_{-}}}+\delta\right)}\left(\varepsilon^{\frac{p_{-}-1}{p_{-}}}+\delta\right)+C\left(e^{C \lambda(\mu+1)\left(\varepsilon^{\frac{p_{-}-1}{p_{-}}}+\delta\right)}-1\right)^{p_{-}-1}\right)
\end{aligned}
$$

when $\delta, \varepsilon$, and $\lambda(\mu+1)\left(\varepsilon^{\left(p_{-}-1\right) / p_{-}}+\delta\right)$ are small, for some positive constant $C$ independent of $\lambda, \mu, \delta, \varepsilon, x$, and $t$. In case $p_{-} \leq 2$ and $\xi_{0} \neq \xi_{1}$, since $\left(\xi_{0}, t_{0}\right) \in \partial B_{\left(\xi_{1}, t_{1}\right)}^{p_{-}}\left(\mu, r_{0}\right)$, we get $\mu\left|t_{1}-t_{0}\right|^{p_{-} /\left(p_{-}-1\right)}<r_{0}$. We choose $\lambda$ large enough so that

$$
\begin{cases}\lambda>\frac{\left(p_{-}-1\right)^{p_{-}-1}}{p_{-}^{p_{-}}\left(r_{0}-\mu\left|t_{1}-t_{0}\right|^{\frac{p_{-}}{p_{-}}}\right)} \sum_{i=1}^{n}\left(\frac{p_{i}}{p_{i}-1}\right)^{p_{i}-1} & \text { if } p_{-}<2 \text { and } \xi_{0} \neq \xi_{1} \\ \lambda>\frac{1}{4\left(r_{0}-\mu\left(t_{1}-t_{0}\right)^{2}\right)}\left(2 \mu\left(t_{1}-t_{0}\right)+\sum_{i=1}^{n}\left(\frac{p_{i}}{p_{i}-1}\right)^{p_{i}-1}\right) & \text { if } p_{-}=2 \text { and } \xi_{0} \neq \xi_{1}\end{cases}
$$

It follows from (3.10), (3.11), and (3.12) that in case $p_{-} \leq 2$ and $\xi_{0} \neq \xi_{1}$, for $\delta$ and $\varepsilon$ small, the function $v_{\lambda, \mu, \delta}$ is a $C^{1}$-solution of the inequality

$$
\frac{\partial v_{\lambda, \mu, \delta}}{\partial t}-\Delta_{\vec{p}} v_{\lambda, \mu, \delta}+f\left(v_{\lambda, \mu, \delta}\right)<0 \quad \text { in } B_{\left(\xi_{0}, t_{0}\right)}^{\vec{p}}\left(\mu, \lambda^{2} \delta, \varepsilon\right)
$$

where $B_{\left(\xi_{0}, t_{0}\right)}^{\vec{p}}\left(\mu, \lambda^{2} \delta, \varepsilon\right)$ is as in (3.9). In case $p_{-}=2$ and $t_{0}=t_{1}+\sqrt{r_{0} / \mu}$, we assume that $\mu>\frac{1}{4 r_{0}}\left(\sum_{i=1}^{n}\left(\frac{p_{i}}{p_{i}-1}\right)^{p_{i}-1}\right)^{2}$, we let $\lambda$ be an arbitrary positive real number, and we also find (3.13) for $\delta$ and $\varepsilon$ small. In case $p_{-}>2$ and $t_{0}>t_{1}$, without assumption on $\lambda$ and $\mu$, we still get (3.13) for $\delta$ and $\varepsilon$ small. Now, we claim that there exists a positive constant $C_{\varepsilon}$ such that $u \geq C_{\varepsilon}$ on $B_{\left(\xi_{1}, t_{1}\right)}^{\vec{p}}\left(\mu, \lambda^{2} \delta, r_{0}\right) \cap \partial B_{\left(\xi_{0}, t_{0}\right)}^{\vec{p}}\left(\mu, \lambda^{2} \delta, \varepsilon\right)$ for $\delta$ small, where $B_{\left(\xi_{1}, t_{1}\right)}^{\vec{p}}\left(\mu, \lambda^{2} \delta, r_{0}\right)$ and $B_{\left(\xi_{0}, t_{0}\right)}^{\vec{p}}\left(\mu, \lambda^{2} \delta, \varepsilon\right)$ are as in (3.9). In order to prove this claim, we proceed by contradiction and assume that there exist a sequence of positive real numbers $\left(\delta_{\alpha}\right)_{\alpha}$ and a sequence of points $\left(\xi_{\alpha}, t_{\alpha}\right)_{\alpha}$ such that $\delta_{\alpha} \rightarrow 0, u\left(\xi_{\alpha}, t_{\alpha}\right) \rightarrow 0$ as $\alpha \rightarrow+\infty$, and $\left(\xi_{\alpha}, t_{\alpha}\right) \in B_{\left(\xi_{1}, t_{1}\right)}^{\vec{p}}\left(\mu, \lambda^{2} \delta_{\alpha}, r_{0}\right) \cap$ 
$\partial B_{\left(\xi_{0}, t_{0}\right)}^{\vec{p}}\left(\mu, \lambda^{2} \delta_{\alpha}, \varepsilon\right)$ for all $\alpha$. Up to a subsequence, we get that $\left(\xi_{\alpha}, t_{\alpha}\right)$ converges to a point $\left(\xi_{\infty}, t_{\infty}\right)$ in $\overline{B_{\left(\xi_{1}, t_{1}\right)}^{p_{-}}\left(\mu, r_{0}\right)} \cap \partial B_{\left(\xi_{0}, t_{0}\right)}^{p_{-}}(\mu, \varepsilon)$, where $B_{\left(\xi_{1}, t_{1}\right)}^{p_{-}}\left(\mu, r_{0}\right)$ and $\partial B_{\left(\xi_{0}, t_{0}\right)}^{p_{-}}(\mu, \varepsilon)$ are as in $(3.1)$ and (3.2). By continuity of the function $u$, we get $u\left(\xi_{\infty}, t_{\infty}\right)=0$, and thus $\left(\xi_{\infty}, t_{\infty}\right)=\left(\xi_{0}, t_{0}\right)$. Since $\left(\xi_{\alpha}, t_{\alpha}\right) \in B_{\left(\xi_{1}, t_{1}\right)}^{\vec{p}}\left(\mu, \lambda^{2} \delta_{\alpha}, r_{0}\right)$ for all $\alpha$ and since $\xi_{1, i}=\xi_{0, i}$ for all $i>n_{-}$, it follows that

$$
\sum_{i=n_{-}+1}^{n}\left(\lambda^{2} \delta_{\alpha}\right)^{\frac{p_{-}-p_{i}}{p_{i}-1}}\left|\xi_{\alpha, i}-\xi_{0, i}\right|^{\frac{p_{i}}{p_{i}-1}}<r_{0}-\mu\left|t_{\alpha}-t_{1}\right|^{\frac{p_{-}}{p_{-}-1}}-\sum_{i=1}^{n_{-}}\left|\xi_{\alpha, i}-\xi_{1, i}\right|^{\frac{p_{-}}{p_{-}-1}}=\mathrm{o}(1)
$$

as $\alpha \rightarrow+\infty$. On the other hand, since $\left(\xi_{\alpha}, t_{\alpha}\right) \in \partial B_{\left(\xi_{0}, t_{0}\right)}^{\vec{p}}\left(\mu, \lambda^{2} \delta_{\alpha}, \varepsilon\right)$ for all $\alpha$, we get

$$
\sum_{i=n_{-}+1}^{n}\left(\lambda^{2} \delta_{\alpha}\right)^{\frac{p_{-}-p_{i}}{p_{i}-1}}\left|\xi_{\alpha, i}-\xi_{0, i}\right|^{\frac{p_{i}}{p_{i}-1}}=\varepsilon-\mu\left|t_{\alpha}-t_{0}\right|^{\frac{p_{-}}{p_{-}-1}}-\sum_{i=1}^{n_{-}}\left|\xi_{\alpha, i}-\xi_{0, i}\right|^{\frac{p_{-}}{p_{-}-1}}=\varepsilon+\mathrm{o}(1)
$$

as $\alpha \rightarrow+\infty$. There is a contradiction between (3.14) and (3.15). This ends the proof of our claim, namely that there exists a positive constant $C_{\varepsilon}$ such that $u \geq C_{\varepsilon}$ on $B_{\left(\xi_{1}, t_{1}\right)}^{\vec{p}}\left(\mu, \lambda^{2} \delta, r_{0}\right) \cap$ $\partial B_{\left(\xi_{0}, t_{0}\right)}^{\vec{p}}\left(\mu, \lambda^{2} \delta, \varepsilon\right)$ for $\delta$ small. Since $v_{\lambda, \mu, \delta} \leq \lambda \delta\left(e^{\lambda r_{0}}-1\right)$ in $B_{\left(\xi_{1}, t_{1}\right)}^{\vec{p}}\left(\mu, \lambda^{2} \delta, r_{0}\right)$ and $v_{\lambda, \mu, \delta} \leq 0$ in $\left(\mathbb{R}^{n} \times \mathbb{R}_{+}\right) \backslash B_{\left(\xi_{1}, t_{1}\right)}^{\vec{p}}\left(\mu, \lambda^{2} \delta, r_{0}\right)$, we then get $v_{\lambda, \mu, \delta} \leq u$ on $\partial B_{\left(\xi_{0}, t_{0}\right)}^{\vec{p}}\left(\mu, \lambda^{2} \delta, \varepsilon\right)$ for $\delta$ small. In particular, there holds $\left(v_{\lambda, \mu, \delta}-u\right)_{+}=0$ on $\partial B_{\left(\xi_{0}, t_{0}\right)}^{\vec{p}}\left(\mu, \lambda^{2} \delta, \varepsilon\right)$, where $\left(v_{\lambda, \mu, \delta}-u\right)_{+}=$ $\max \left(v_{\lambda, \mu, \delta}-u, 0\right)$. Testing (1.8) and (3.13) against $\left(v_{\lambda, \mu, \delta}-u\right)_{+}$on $B_{\left(\xi_{0}, t_{0}\right)}^{\vec{p}}\left(\mu, \lambda^{2} \delta, \varepsilon\right)$ (up to an approximation in terms of Steklov averages, see, for instance, DiBenedetto [15]), we get

$$
\begin{aligned}
\frac{1}{2} \int_{W_{\lambda, \mu, \delta, \varepsilon, t}}\left|v_{\lambda, \mu, \delta}-u\right|^{2} d x & \\
+\sum_{i=1}^{n} \int_{0}^{t} \int_{W_{\lambda, \mu, \delta, \varepsilon, s}} & \left(\left|\frac{\partial v_{\lambda, \mu, \delta}}{\partial x_{i}}\right|^{p_{i}-2} \frac{\partial v_{\lambda, \mu, \delta}}{\partial x_{i}}-\left|\frac{\partial u}{\partial x_{i}}\right|^{p_{i}-2} \frac{\partial u}{\partial x_{i}}\right)\left(\frac{\partial v_{\lambda, \mu, \delta}}{\partial x_{i}}-\frac{\partial u}{\partial x_{i}}\right) d x d s \\
& \quad+\int_{0}^{t} \int_{W_{\lambda, \mu, \delta, \varepsilon, s}}\left(f\left(v_{\lambda, \mu, \delta}\right)-f(u)\right)\left(v_{\lambda, \mu, \delta}-u\right) d x d s \leq 0
\end{aligned}
$$

for all real numbers $t$ in $(0, T)$, where

$$
W_{\lambda, \mu, \delta, \varepsilon, t}=\left\{x \in \mathbb{R}^{n} ; \quad(x, t) \in B_{\left(\xi_{0}, t_{0}\right)}^{\vec{p}}\left(\mu, \lambda^{2} \delta, \varepsilon\right) \quad \text { and } \quad v_{\lambda, \mu, \delta}(x, t)>u(x, t)\right\} .
$$

Since the function $f$ is nondecreasing, it follows from (3.16) that for any real number $t$ in $(0, T)$, there holds

$$
\int_{W_{\lambda, \mu, \delta, \varepsilon, t}}\left|v_{\lambda, \mu, \delta}-u\right|^{2} d x=0 .
$$

We then get $\left|W_{\lambda, \mu, \delta, \varepsilon, t}\right|=0$, i.e. $v_{\lambda, \mu, \delta} \leq u$ in $B_{\left(\xi_{0}, t_{0}\right)}^{\vec{p}}\left(\mu, \lambda^{2} \delta, \varepsilon\right)$. In particular, we get $u\left(\xi_{0}, t_{0}\right) \geq$ $v_{\lambda, \mu, \delta}\left(\xi_{0}, t_{0}\right)>0$. There is a contradiction. This ends the proof of Lemma 3.1.

Now, we can prove Theorem 1.2 by using Lemma 3.1 .

Proof of Theorem 1.2. To begin with, we assume that $p_{-} \leq 2$ and prove that the function $u$ is identically zero on the set $\Omega_{0} \times\left\{t_{0}\right\}$, where $\Omega_{0}$ is as in (1.6). We let $P$ be the set of points $(x, t)$ in $\Omega \times(0, T)$ such that $u(x, t)>0$. We proceed by contradiction and assume that $P \cap\left(\Omega_{0} \times\left\{t_{0}\right\}\right)$ is not empty. In a similar way as in the proof of Theorem 1.1, we can choose a positive real number $r_{0}$ and two points $\zeta_{0}=\left(\zeta_{0,1}, \ldots, \zeta_{0, n}\right)$ and $\xi_{1}=\left(\xi_{1,1}, \ldots, \xi_{1, n}\right)$ 
in $\Omega_{0}$ such that $u\left(\zeta_{0}, t_{0}\right)=0, \zeta_{0} \in \partial B_{\xi_{1}}^{p_{-}}\left(r_{0}\right)$, and $B_{\xi_{1}}^{p_{-}}\left(r_{0}\right) \times\left\{t_{0}\right\} \subset P$, where $B_{\xi_{1}}^{p_{-}}\left(r_{0}\right)$ and $\partial B_{\xi_{1}}^{p_{-}}\left(r_{0}\right)$ are as in $(2.2)$ and $(2.3)$. We let $h:[0,1] \rightarrow \mathbb{R}_{+}$be defined by

$$
h(\delta)=\inf _{(x, t) \in\left(\Omega_{0} \times(0, T)\right) \backslash P}\left(\sum_{i=1}^{n_{-}}\left|x_{i}-\delta \xi_{1, i}-(1-\delta) \zeta_{0, i}\right|^{\frac{p_{-}}{p_{-}-1}}+\left|t-t_{0}\right|^{\frac{p_{-}}{p_{-}-1}}\right) .
$$

Since $u\left(\zeta_{0}, t_{0}\right)=0$, we get $h(\delta) \rightarrow 0$ as $\delta \rightarrow 0$. In particular, we get $\partial B_{\left(\delta \xi_{1}+(1-\delta) \zeta_{0}, t_{0}\right)}^{p_{-}}(1, h(\delta)) \subset$ $\Omega_{0} \times(0, T)$ and $B_{\left(\delta \xi_{1}+(1-\delta) \zeta_{0}, t_{0}\right)}^{p_{-}}(1, h(\delta)) \subset P$ for $\delta$ small, where $B_{\left(\delta \xi_{1}+(1-\delta) \zeta_{0}, t_{0}\right)}^{p_{-}}(1, h(\delta))$ and $\partial B_{\left(\delta \xi_{1}+(1-\delta) \zeta_{0}, t_{0}\right)}^{p_{-}}(1, h(\delta))$ are as in (3.1) and (3.2). Since $P$ is open, it follows that for $\delta$ small, the infimum in $(3.17)$ is achieved, i.e. there exists a point $\left(\zeta_{\delta}, t_{\delta}\right)$ on $\partial B_{\left(\delta \xi_{1}+(1-\delta) \zeta_{0}, t_{0}\right)}^{p_{-}}(1, h(\delta))$ such that $u\left(\zeta_{\delta}, t_{\delta}\right)=0$. By Lemma 3.1, we get $\zeta_{\delta}=\delta \xi_{1}+(1-\delta) \zeta_{0}$, and thus $h(\delta)=$ $\left|t_{\delta}-t_{0}\right|^{p_{-} /\left(p_{-}-1\right)}$ for $\delta$ small. It follows from (3.17) that for $\delta_{1}$ and $\delta_{2}$ small, there holds

$$
h\left(\delta_{1}\right) \leq \sum_{i=1}^{n_{-}}\left|\xi_{1, i}-\zeta_{0, i}\right|^{\frac{p_{-}}{p_{-}-1}}\left|\delta_{2}-\delta_{1}\right|^{\frac{p_{-}}{p_{-}-1}}+h\left(\delta_{2}\right) .
$$

In particular, for $\delta$ small, the function $h$ is differentiable and $h^{\prime}=0$ on $[0, \delta]$. It follows that the function $h$ is constant on $[0, \delta]$. Since $h(0)=0$, we then get $h=0$ on $[0, \delta]$, i.e. $u=0$ on $\left[\delta \xi_{1}+(1-\delta) \zeta_{0}, \zeta_{0}\right] \times\left\{t_{0}\right\}$. There is a contradiction. This ends the proof of the first part of Theorem 1.2. Now, we assume that $p_{-} \geq 2$, and we prove that the function $u$ is identically zero on the set $\left\{\xi_{0}\right\} \times\left(0, t_{0}\right]$. We proceed by contradiction and assume that there exists a real number $t_{1}$ in $\left(0, t_{0}\right)$ such that $u\left(\xi_{0}, t_{1}\right)>0$. Since $P$ is open, we get $B_{\left(\xi_{0}, t_{1}\right)}^{p_{-}}(\mu, \varepsilon) \subset P$ for $\mu$ large and $\varepsilon$ small, where $B_{\left(\xi_{0}, t_{1}\right)}^{p_{-}}(\mu, \varepsilon)$ is as in (3.1). We may assume that the real number $\mu$ is large enough so that $\mu>\frac{1}{2 \varepsilon} \sum_{i=1}^{n}\left(\frac{p_{i}}{p_{i}-1}\right)^{p_{i}-1}$. Increasing, if necessary, the real number $t_{1}$, since $P$ is open, we may assume that

$$
t_{1}=\sup \left\{t \in\left(0, t_{0}\right) ; \quad B_{\left(\xi_{0}, t_{1}\right)}^{p_{-}}(\mu, \varepsilon) \subset P\right\} .
$$

It follows that there exists a point $\left(\xi_{2}, t_{2}\right)$ on $\partial B_{\left(\xi_{0}, t_{1}\right)}^{p_{-}}(\mu, \varepsilon)$ such that $t_{2}>t_{1}$ and $u\left(\xi_{2}, t_{2}\right)=0$. We get a contradiction with Lemma 3.1. This ends the proof of Theorem 1.2.

Acknowledgments: The author was partially supported by the ANR grant ANR-08-BLAN0335-01.

\section{REFERENCES}

[1] C. O. Alves and A. El Hamidi, Existence of solution for a anisotropic equation with critical exponent, Differential Integral Equations 21 (2008), no. 1, 25-40.

[2] S. Antontsev and M. Chipot, Anisotropic equations: uniqueness and existence results, Differential Integral Equations 21 (2008), no. 5-6, 401-419.

[3] S. Antontsev, J. I. Díaz, and S. Shmarev, Energy methods for free boundary problems: Applications to nonlinear PDEs and fluid mechanics, Progress in Nonlinear Differential Equations and their Applications, vol. 48, Birkhäuser, Boston, 2002.

[4] S. Antontsev and S. Shmarev, Elliptic equations with anisotropic nonlinearity and nonstandard growth conditions, Handbook of Differential Equations: Stationary Partial Differential Equations, vol. 3, Elsevier, Amsterdam, 2006.

[5] - Parabolic equations with anisotropic nonstandard growth conditions, Internat. Ser. Numer. Math., vol. 154, Birkhäuser, Basel, 2007, pp. 33-44.

[6] Extinction of solutions of parabolic equations with variable anisotropic nonlinearities, Trudy Mat. Inst. Steklov. 261 (2008), 16-25 (Russian); English transl., Proc. Steklov Inst. Math. 261 (2008), 1-11. 
[7] _ Vanishing solutions of anisotropic parabolic equations with variable nonlinearity, J. Math. Anal. Appl. 361 (2010), no. 2, 371-391.

[8] __ Localization of solutions of anisotropic parabolic equations, Nonlinear Anal. 71 (2009), no. 12, 725-737.

[9] J. Bear, Dynamics of Fluids in Porous Media, American Elsevier, New York, 1972.

[10] M. Bendahmane and K. H. Karlsen, Renormalized solutions of an anisotropic reaction-diffusion-advection system with $L^{1}$ data, Commun. Pure Appl. Anal. 5 (2006), no. 4, 733-762.

[11] _ Nonlinear anisotropic elliptic and parabolic equations in $\mathbb{R}^{N}$ with advection and lower order terms and locally integrable data, Potential Anal. 22 (2005), no. 3, 207-227.

[12] M. Bendahmane, M. Langlais, and M. Saad, On some anisotropic reaction-diffusion systems with $L^{1}$-data modeling the propagation of an epidemic disease, Nonlinear Anal. 54 (2003), no. 4, 617-636.

[13] A. Cianchi, Symmetrization in anisotropic elliptic problems, Comm. Partial Differential Equations 32 (2007), no. 4-6, 693-717.

[14] L. D'Ambrosio, Liouville theorems for anisotropic quasilinear inequalities, Nonlinear Anal. 70 (2009), no. 8, 2855-2869.

[15] E. DiBenedetto, Degenerate parabolic equations, Universitext, Springer-Verlag, New York, 1993.

[16] A. Di Castro, Existence and regularity results for anisotropic elliptic problems, Adv. Nonlin. Stud. 9 (2009), 367-393.

[17] A. Di Castro and E. Montefusco, Nonlinear eigenvalues for anisotropic quasilinear degenerate elliptic equations, Nonlinear Anal. 70 (2009), no. 11, 4093-4105.

[18] A. Di Castro, M. Pérez-Llanos, and J. M. Urbano, Limits of anisotropic and degenerate elliptic problems, Commun. Pure Appl. Anal. (2011). To appear.

[19] A. El Hamidi and J.-M. Rakotoson, On a perturbed anisotropic equation with a critical exponent, Ricerche Mat. 55 (2006), no. 1, 55-69.

[20] __ Extremal functions for the anisotropic Sobolev inequalities, Ann. Inst. H. Poincare Anal. Non Linéaire 24 (2007), no. 5, 741-756.

[21] A. El Hamidi and J. Vétois, Sharp Sobolev asymptotics for critical anisotropic equations, Arch. Ration. Mech. Anal. 192 (2009), no. 1, 1-36.

[22] R. Fortini, D. Mugnai, and P. Pucci, Maximum principles for anisotropic elliptic inequalities, Nonlinear Anal. 70 (2009), no. 8, 2917-2929.

[23] I. Fragalà, F. Gazzola, and B. Kawohl, Existence and nonexistence results for anisotropic quasilinear elliptic equations, Ann. Inst. H. Poincaré Anal. Non Linéaire 21 (2004), no. 5, 715-734.

[24] I. Fragalà, F. Gazzola, and G. Lieberman, Regularity and nonexistence results for anisotropic quasilinear elliptic equations in convex domains, Discrete Contin. Dyn. Syst. suppl. (2005), 280-286.

[25] J. García-Melián, J. D. Rossi, and J. C. Sabina de Lis, Large solutions to an anisotropic quasilinear elliptic problem, Ann. Mat. Pura Appl. (4) 189 (2010), no. 4, 689-712.

[26] M. Giaquinta, Growth conditions and regularity, a counterexample, Manuscripta Math. 59 (1987), no. 2, 245-248.

[27] E. Hopf, Elementare Bemerkungen über die lösungen partielle differentialgleichungen zweiter ordnung vom elliptischen typus, Sitz. Preuss. Akad. Wiss. 19 (1927), 147-152.

[28] F. Q. Li, Anisotropic elliptic equations in $L^{m}$, J. Convex Anal. 8 (2001), no. 2, 417-422.

[29] G. M. Lieberman, Gradient estimates for a new class of degenerate elliptic and parabolic equations, Ann. Scuola Norm. Sup. Pisa Cl. Sci. (4) 21 (1994), no. 4, 497-522.

[30] _ Gradient estimates for anisotropic elliptic equations, Adv. Differential Equations 10 (2005), no. 7, 767-812.

[31] P. Marcellini, Un exemple de solution discontinue d'un problème variationnel dans le cas scalaire, Preprint Istituto Matematico U. Dini 11 (1987).

[32] _ Regularity and existence of solutions of elliptic equations with p, q-growth conditions, J. Differential Equations 90 (1991), no. 1, 1-30.

[33] A. Mercaldo, J. D. Rossi, S. Segura de León, and C. Trombetti, Anisotropic p, q-Laplacian equations when $p$ goes to 1, Nonlinear Anal. 73 (2010), no. 11, 3546-3560.

[34] M. Mihăilescu, P. Pucci, and V. Rădulescu, Eigenvalue problems for anisotropic quasilinear elliptic equations with variable exponent, J. Math. Anal. Appl. 340 (2008), no. 1, 687-698.

[35] M. Mihăilescu, V. Rădulescu, and S. Tersian, Eigenvalue problems for anisotropic discrete boundary value problem, Journal of Difference Equations and Applications 15 (2009), no. 6, 557-567. 
[36] Y. V. Namlyeyeva, A. E. Shishkov, and I. I. Skrypnik, Isolated singularities of solutions of quasilinear anisotropic elliptic equations, Adv. Nonlinear Stud. 6 (2006), no. 4, 617-641.

[37] B. Nazaret, Principe de maximum strict pour un opérateur quasi linéaire, C. R. Acad. Sci. Paris Sér. I Math. 333 (2001), no. 2, 97-102 (French).

[38] L. Nirenberg, A strong maximum principle for parabolic equations, Comm. Pure Appl. Math. 6 (1953), $167-177$.

[39] I. Peral Alonso, Multiplicity of solutions for the p-Laplacian, Lecture notes of the second international school in functional analysis and applications to differential equations (I.C.T.P., Trieste, 1997).

[40] M. Pérez-Llanos and J. D. Rossi, An anisotropic infinity Laplacian obtained as the limit of the anisotropic $(p, q)$-Laplacian, Commun. Contemp. Math. (2011). To appear.

[41] M. H. Protter and H. F. Weinberger, Maximum principles in differential equations, Prentice-Hall Inc., Englewood Cliffs, N.J., 1967.

[42] I. I. Skrypnik, Removability of an isolated singularity for anisotropic elliptic equations with absorption, Math. Sb. 199 (2008), no. 7, 1033-1050.

[43] A. S. Tersenov and A. S. Tersenov, The problem of Dirichlet for anisotropic quasilinear degenerate elliptic equations, J. Differential Equations 235 (2007), no. 2, 376-396.

[44] J. L. Vázquez, A strong maximum principle for some quasilinear elliptic equations, Appl. Math. Optim. 12 (1984), no. 3, 191-202.

[45] J. Vétois, A priori estimates for solutions of anisotropic elliptic equations, Nonlinear Anal. 71 (2009), no. 9, 3881-3905.

[46] _ Asymptotic stability, convexity, and Lipschitz regularity of domains in the anisotropic regime, Commun. Contemp. Math. 12 (2010), no. 1, 35-53.

[47] Existence and regularity for critical anisotropic equations with critical directions, Adv. Differential Equations 16 (2011), no. 1/2,61-83.

[48] _ The blow-up of critical anistropic equations with critical directions, Nonlinear Differ. Equ. Appl. 18 (2011), no. 2, 173-197.

[49] Qihu Zhang, A strong maximum principle for differential equations with nonstandard $p(x)$-growth conditions, J. Math. Anal. Appl. 312 (2005), no. 1, 24-32.

Jérôme Vétois, Université de Nice - Sophia Antipolis, Laboratoire J.-A. Dieudonné, UmR CNRS-UnS 6621, Parc Valrose, 06108 Nice Cedex 2, France

E-mail address: vetois@unice.fr 